\title{
A multi-band shunt hybrid active filter with reduced sensor count
}

\author{
S SURENDRA KUMAR $^{1}$ and PARTHA SARATHI SENSARMA ${ }^{2}$ \\ ${ }^{1}$ Samsung India Software Operations Pvt. Ltd. Byrasandra, Bangalore 560093 \\ ${ }^{2}$ Department of Electrical Engineering, Indian Institute of Technology, \\ Kanpur 208016 \\ e-mail: surendra.ks@samsung.com; sensarma@iitk.ac.in
}

\begin{abstract}
A Shunt Hybrid Active Filter (SHAF) is an attractive option for realizing low-cost harmonic compensation solutions. This paper proposes a SHAF with multiple harmonic compensation capability using a single Voltage Source Inverter and reduced sensor count. This strategy is apt for harmonic filtering solutions where low cost is the exclusive priority. In this paper, a new estimation approach is proposed to obviate requirement of a large number of sensors. Multiple Synchronous Reference Frames (MSRF) and low pass filters are used to measure $5^{\text {th }}$ and $7^{\text {th }}$ harmonic components separately from load as well as filter currents. Individual current controllers are designed for the $5^{\text {th }}$ and $7^{\text {th }}$ harmonic currents. Control is realized in the synchronously rotating, orthogonal $(d q)$ reference frame. Performance of the controller is validated through simulation, using realistic plant and controller models. Experimental results are provided to corroborate the analytical and simulation results.
\end{abstract}

Keywords. Hybrid active filter; harmonic extraction; multiple synchronous reference frames; power quality.

\section{Introduction}

With electronic equipment pervading most spheres of human activity, usage of uncontrolled or semi-controlled rectifiers has increased manifold. These rectifiers, which are rugged and inexpensive, convert alternating (ac) power from low voltage $(220 \mathrm{~V} / 110 \mathrm{~V})$ electrical utility networks to unidirectional $(\mathrm{dc})$ voltage, required for energizing electronic equipment. Despite their obvious merits, all such rectifiers draw disproportionately large harmonic currents which flow through the network impedances and cause harmonic voltages to appear in the network neighbourhood. Harmonic voltages have been reported (Dugan et al 2003) to cause severe overheating of electromagnetic equipment including transformers and induction motors, as well as speeding up of energy meters.

A common method to prevent appearance of harmonic voltages involves diverting harmonic currents away from the power network. This method, termed harmonic filtering, commonly requires a filter equipment connected to the Point of Common Coupling (PCC), in shunt with 

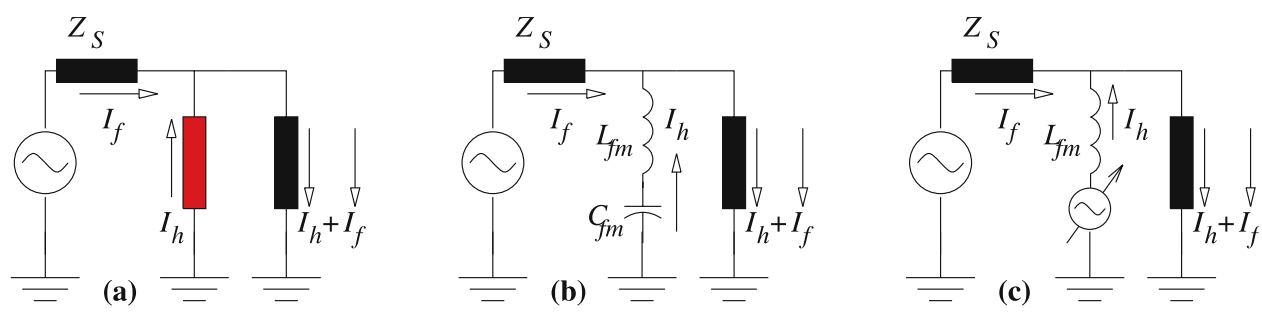

Figure 1. Basic harmonic filtering strategy: (a) Concept, (b) Passive filter, (c) Active filter.

the rectifier as shown in figure 1a. Harmonic filters were traditionally configured with series resonant networks using passive elements, shown in figure $1 \mathrm{~b}$. Proper selection of passive elements ensures accurate match of the resonant frequency with the rectifier current harmonic frequency. However, as the passive elements $\left(C_{f m}, L_{f m}\right)$ tend to drift under external influence, the filtering performance deteriorates. Also, these are prone to resonances with the power network, usually with disastrous consequences.

Drawbacks of the passive filter are overcome by active filters, shown in figure 1c. Active filters comprise a voltage source inverter (VSI) connected in shunt with the power line through a series inductor. In the figure, the VSI is shown modelled as a controlled voltage source. With a suitable closed-loop controller regulating this effective voltage source, the active filter is made to inject an identical harmonic current drawn by the load, but in opposite phase. As these require large VSI ratings, active filters are expensive, hence sparingly deployed.

Shunt Hybrid Active Filters (SHAF), consisting of a VSI and passive filter in series, have been extensively reported (Fujita \& Akagi 1991); (Cheng et al 1999); (Srianthumrong \& Akagi 2003); (Inunza \& Akagi 2005); (Chen et al 2004). This combination is placed in shunt with a non-linear load, as shown in figure 2 . This topology overcomes the disadvantages of filter overload and system resonance with a passive filter. Also, it results in significant reduction in the VSI rating, hence cost. This reduction happens because the VSI is not required to overcome the fundamental component of the PCC voltage. Various control strategies have been proposed for the control of these filters and a comprehensive review is presented in (Akagi 1996). The simpler ones among these schemes use a resistor-emulation approach, based on either PCC voltage or source current sensing (Akagi 1997). But these schemes are prone to instability (Peng 1998), especially with strong grids, and closed-loop stability is more suspect than feedforward methods based on load current measurement (Sensarma et al 2000). A major bone of contention with the SHAF, however, is the problem of controlling the dc bus voltage of the VSI. Since the SHAF is ideally not capable of injecting any controlled fundamental current, control of the VSI dc bus voltage assumes critical importance. A realizable solution involves

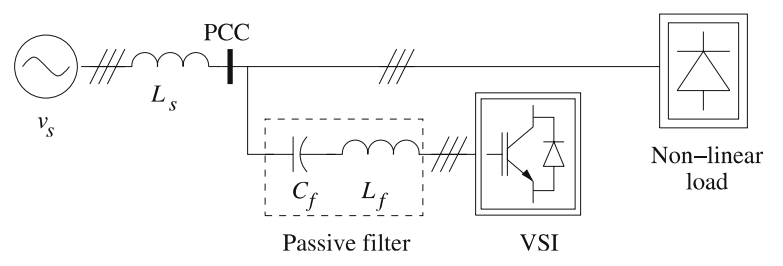

Figure 2. Schematic of basic shunt hybrid active filter. 


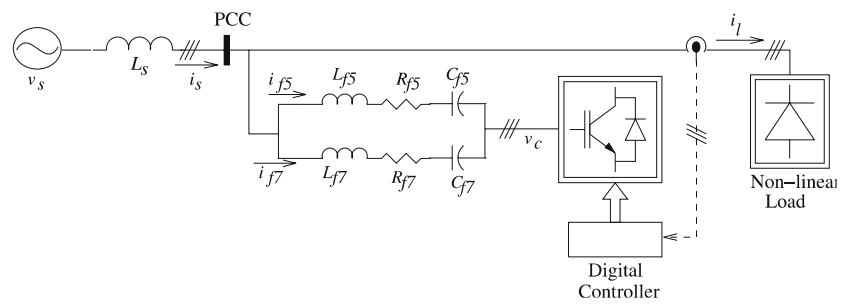

Figure 3. Schematic of a multiband shunt hybrid active filter.

use of an additional low power transformer-VSI combination to control the VSI dc bus voltage (Rastogi et al 1995). This approach effectively decouples the problems of harmonic filtering and dc bus voltage control. Individual solutions to each of these problems can be seamlessly superposed for reliable operation of the complete system.

In its basic form, one SHAF branch can compensate for only one harmonic frequency. As non-linear loads usually inject several dominant harmonic currents, this requires multiple VSI$\mathrm{s}$ for satisfactory harmonic compensation. VSI-s being the most cost-intensive component of the SHAF, this scheme compromises on the basic cost advantage.

A separate class of solutions (Longhui et al 2007); (Zhai et al 2006); (Routimo et al 2004); (Chen et al 2004) is aimed at damping of harmonic resonances using a hybrid active filter. However, in all these cases, the VSI and the passive filter are individually connected in shunt to the PCC. Although successful in their basic objective, this class of solution requires a VSI of large rating, thereby losing the cost advantage.

The possibility of using a single VSI in series with multiple passive filter branches has been reported in Park et al (2000). The general circuit schematic of this approach is shown in figure 3. This is significantly less expensive in comparison with the multiple VSI scheme owing to use of a single VSI of comparable rating. However, the reported approach requires measurement of the filter capacitor $\left(C_{f m}\right)$ voltages for effective control. This implies additional complexity and cost as well as reduced reliability of an otherwise low-cost solution, especially when the number of passive filter branches increase.

In this paper, a multi-band SHAF is considered, in which two passive filter branches, tuned at $5^{\text {th }}$ and $7^{\text {th }}$ harmonic frequencies, are connected in series with the VSI. Design of passive filter elements is detailed. Apart from harmonic tuning, the passive filter impedances are designed for inductive behaviour at the switching frequency, for filtering switching ripples in VSI output. The controller is designed using frequency domain approach while disturbance rejection is achieved with feed-forward compensation in time domain. A new estimation scheme is proposed which enables realization of the control scheme using measurements of PCC voltages, load currents and VSI currents. Since three-wire systems are considered, only two phases of the above variables are measured which drastically reduces sensor count. Since these measurement overheads do not scale with the number of passive filter branches, the cost advantage increasingly improves as more frequencies are added to the filtering band. Low-cost, reliable filtering performance being the focal objective of this paper, the issue of dc bus voltage control has not been addressed here. All analytical control design is validated using simulation, on MATLAB-SIMULINK platform. Multi-rate sampling is used to model the plant (fast sample-rate) and the controller (slow sample-rate). Exact switching functions have been used in the simulation model, though switch losses are not considered. Experimental results on full-scale hardware are provided to corroborate the analytical and simulation results. 
Table 1. Passive filter parameters.

\begin{tabular}{lccccc}
\hline$L_{f 5}$ & $R_{f 5}$ & $C_{f 5}$ & $L_{f 7}$ & $R_{f 7}$ & $C_{f 7}$ \\
\hline $40.5 \mathrm{mH}$ & $1 \Omega$ & $10.0 \mu \mathrm{F}$ & $20.7 \mathrm{mH}$ & $0.41 \Omega$ & $10.0 \mu \mathrm{F}$ \\
$(0.459 \mathrm{pu})$ & $(0.0361 \mathrm{pu})$ & $(0.0869 \mathrm{pu})$ & $(0.235 \mathrm{pu})$ & $(0.0148 \mathrm{pu})$ & $(0.0869 \mathrm{pu})$ \\
\hline
\end{tabular}

\section{Design of passive filter}

A series resonant filter circuit is shown in figure $1 \mathrm{~b}$. Impedance $\left(Z_{f m}\right)$ and nominal resonant frequency $\left(\omega_{m}\right)$ of the $m^{\text {th }}$ tuned passive branch $(m=5,7)$ are

$$
Z_{f m}(s)=\frac{s^{2} L_{f m} C_{f m}+1}{s C_{f m}} ; \omega_{m}=\sqrt{\frac{1}{L_{f m} C_{f m}}}, \text { where } \omega_{m}=m \cdot 2 \pi f .
$$

Using (1), specific filter components are designed which is detailed further below.

\subsection{Selection of $C_{f m}$}

The choice of $C_{f m}$ is decided by the following factors. A high value reduces impedance at fundamental frequency resulting in passage of fundamental frequency $(f)$ current, which is highly undesirable. Also a low value results in a bulky inductor in the resonant branch, which in turn increases its cost. The optimal value of the filter capacitor is found to be $10 \mu \mathrm{F}$. All relevant filter parameters are listed in table $1 . R_{f 5}$ and $R_{f 7}$ are the internal resistances of $L_{f 5}$ and $L_{f 7}$. Per-unit values are derived with rated load parameters as base values.

\subsection{Impedance characteristic of passive filter circuit}

Figure 4 shows the Bode plot (magnitude and phase) of the passive branch, for the filter parameters chosen. It shows the fundamental component and the switching harmonics $(10 \mathrm{kHz}$

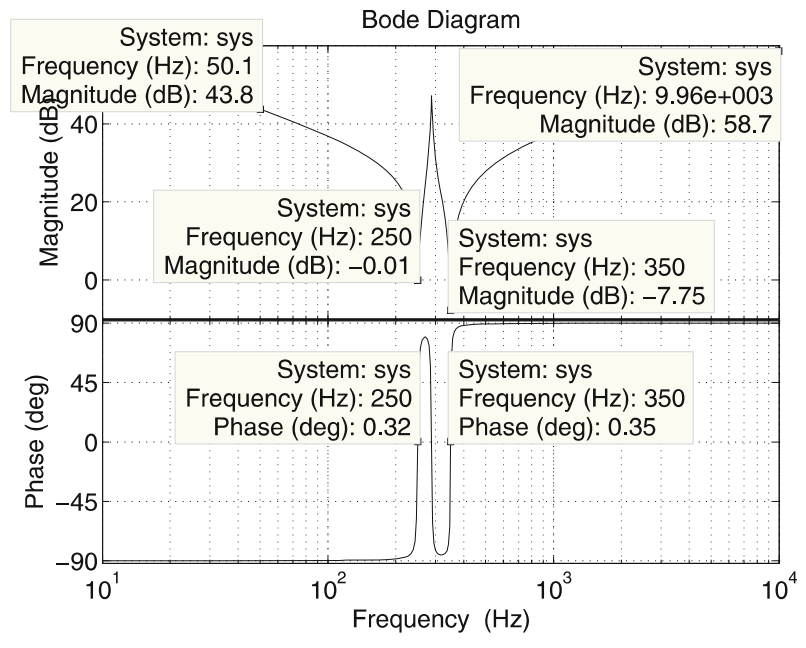

Figure 4. Bode plot for passive filter impedance, $Z_{f}(s)$, in $\mathrm{dB} \Omega$. 


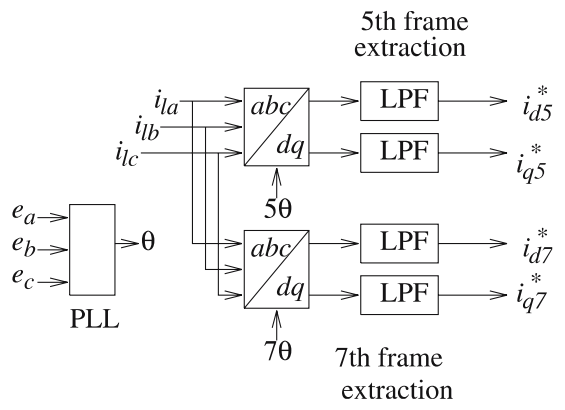

Figure 5. Block diagram showing reference current generation using MSRF.

and multiples) are blocked by the passive filter, whereas at other desired $5^{\text {th }}$ and $7^{\text {th }}$ harmonic frequencies the filter offers low impedance. Switching harmonics of the active filter output are filtered by the passive filter impedance, which gives more than $55 \mathrm{~dB}$ attenuation at $10 \mathrm{kHz}$. Hence only the required load harmonic currents will pass through the filter branches while fundamental currents will be largely blocked. This results in a great reduction of the required VSI rating, as compared to a pure Active Filter.

\section{Harmonic extraction}

In this paper, only $5^{\text {th }}$ and $7^{\text {th }}$ harmonic components are sought to be compensated. These are extracted from a measurement of the load current using Multiple Synchronous Reference Frames (MSRF) and low pass filters (Park et al 2000); (Bhattacharya et al 1991). This method uses Park's transformation on two-phase input quantities, $\left[\begin{array}{ll}X_{\alpha} & X_{\beta}\end{array}\right]^{\prime}$, whose projections on a reference frame, rotating at an angular speed $\omega$, are $\left[\begin{array}{ll}X_{d} & X_{q}\end{array}\right]^{\prime}$. Mathematically, this transformation is expressed as

$$
\left[\begin{array}{l}
X_{d} \\
X_{q}
\end{array}\right]=[T]\left[\begin{array}{l}
X_{\alpha} \\
X_{\beta}
\end{array}\right], \quad \text { where }[T]=\left[\begin{array}{cc}
\cos \omega t & -\sin \omega t \\
\sin \omega t & \cos \omega t
\end{array}\right] .
$$

If the input is a set of balanced sinusoids with arbitrary frequency $\omega_{1}$, then the input and output quantities are

$$
\left[\begin{array}{l}
X_{\alpha} \\
X_{\beta}
\end{array}\right]=\sqrt{2} X\left[\begin{array}{c}
\cos \left(\omega_{1} t+\phi\right) \\
\sin \left(\omega_{1} t+\phi\right)
\end{array}\right] \text { and }\left[\begin{array}{l}
X_{d} \\
X_{q}
\end{array}\right]=\sqrt{2} X\left[\begin{array}{c}
\cos \left(\overline{\omega_{1} \sim \omega} t+\phi\right) \\
\sin \left(\overline{\omega_{1} \sim \omega} t+\phi\right)
\end{array}\right]
$$

Usually the transform outputs $\left(X_{d}, X_{q}\right)$ are also sinusoidal ac quantities, except for $\omega_{1}=$ $\omega$, when the outputs become constant. So, the measured load currents are projected on a reference frame rotating at a harmonic frequency $\omega_{h}$. Only the specific harmonic at frequency $\omega_{h}(=2 \pi m f)$ gets transformed to a dc quantity, which is extracted using a low-pass filter. Unit vectors $(\cos \omega t, \sin \omega t)$ of the transformation $[T]$ are obtained from a PLL (Kaura \& Blasko 1997). Schematic of the harmonic extraction process is shown in figure 5.

\section{System model}

Per-phase harmonic equivalent circuit of the SHAF is shown in figure 6. $v_{c}$ is the VSI output voltage and $V_{p c c m}$ is the harmonic component of the PCC voltage. If the PCC voltage is purely 


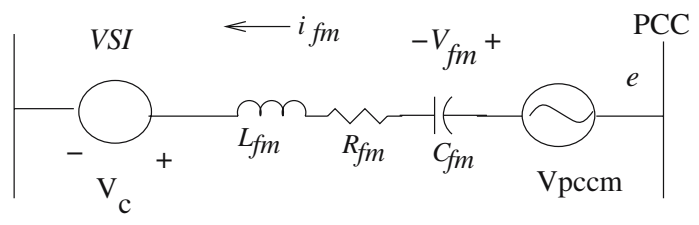

Figure 6. Shunt hybrid active filter equivalent circuit for harmonics.

sinusoidal then $V_{p c c m}=0$. Then, from figure 6 , the per-phase dynamic equation for the filter current is

$$
v_{c}+L_{f m} \frac{d i_{f m}}{d t}+i_{f m} R_{f m}+v_{f m}=0
$$

Transforming (4) using $m^{\text {th }}$ order SRF frame (reference frame rotating at angular speed of

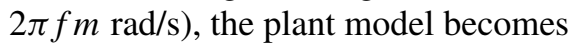

$$
\frac{d}{d t}\left[\begin{array}{c}
i_{f m d}^{m} \\
i_{f m q}^{m}
\end{array}\right]=\left[\begin{array}{cc}
-\frac{R_{f m}}{L_{f m}} & -m \omega \\
m \omega & -\frac{R_{f m}}{L_{f m}}
\end{array}\right]\left[\begin{array}{c}
i_{f m d}^{m} \\
i_{f m q}^{m}
\end{array}\right]-\frac{1}{L_{f m}}\left\{\left[\begin{array}{c}
v_{c d}^{m} \\
v_{c q}^{m}
\end{array}\right]-\left[\begin{array}{c}
v_{f m d}^{m} \\
v_{f m q}^{m}
\end{array}\right]\right\},
$$

where superscripts denote the SRF order. In (5), the non-zero off-diagonal elements of the system matrix (square) indicate dynamic coupling between the $d$ and $q$ axes. Control inputs to the system are $v_{c d / q}^{m}$ and the disturbance inputs are $v_{f m d / q}^{m}$.

\section{Current controller design}

Individual current controllers are designed for the $5^{\text {th }}$ and $7^{\text {th }}$ harmonic currents. The extracted load harmonic currents are used as reference commands for the individual current control loops. Control is realized in the MSRF domain, which simplifies an inherently tracking problem to one of regulator design.

\subsection{Controller for $5^{\text {th }}$ and $7^{\text {th }}$ harmonic currents}

The control objective is to ensure accurate command following while minimizing the effects of disturbance inputs on filter performance. This is proposed to be achieved by using sequential vector decoupling and feed-forward compensation using PCC voltage measurements. Defining

$$
\begin{aligned}
u_{m d 1}^{m} & =-v_{c d}^{m}-v_{f m d}^{m} \\
u_{m q 1}^{m} & =-v_{c q}^{m}-v_{f m q}^{m} \\
u_{m d}^{m} & =u_{d m 1}-m \omega L_{f m} i_{f m q}^{m} \\
u_{m q}^{m} & =u_{q m 1}+m \omega L_{f m} i_{f m d}^{m} .
\end{aligned}
$$

and substituting in (5) gives

$$
L_{f m} \frac{d}{d t}\left[\begin{array}{c}
i_{f m d}^{m} \\
i_{f m q}^{m}
\end{array}\right]+R_{f m}\left[\begin{array}{c}
i_{f m d}^{m} \\
i_{f m q}^{m}
\end{array}\right]=\left[\begin{array}{c}
u_{m d}^{m} \\
u_{m q}^{m}
\end{array}\right] .
$$


The effective plant described by (7) consists of two decoupled, first-order, single input single output (SISO) systems. Consequently, controller design for the plant is greatly simplified. A PI controller, $H_{i}(s)$, of the following form is considered.

$$
H_{i}(s)=K_{p}\left(\frac{s+\omega_{i}}{s}\right)
$$

The gain $K_{p}$ of the PI controller is selected so as to achieve the required settling time. Loop transfer function, $G_{m i}(s) H_{m i}(s)$, for the $m^{\text {th }}$ order SRF is therefore

$$
G_{m i}(s) H_{m i}(s)=\frac{K_{p}\left(s+\omega_{i}\right)}{s\left(R_{f m}+s L_{f m}\right)\left(1+s T_{s}\right)}, \quad m=5,7,
$$

where $T_{s}$ is the sampling delay. Since only the total VSI line current is measured, the individual passive filter branch currents are therefore estimated. The underlying approximation in the proposed estimation process is that the $m^{\text {th }}$ harmonic current flows exclusively through the $m^{\text {th }}$ tuned filter branch. Although not strictly correct, it is still a close approximation since the passive filter parameters are selected to impart much higher impedances to all except the tuning frequency.

In (6), $v_{f m d}^{m}$ and $v_{f m q}^{m}$ are measurements of the filter capacitor voltages. In the control law, these signals are essential for disturbance rejection and axis de-coupling. Direct physical measurement would involve using a voltage sensor across each capacitor in each of the phases of every passive tuned branch. This would imply additional complexity and cost as well as reduced reliability. In this paper, an estimation technique is proposed to overcome this difficulty. This is discussed below.

\subsection{Capacitor voltage estimation}

In the $m^{\text {th }}$ tuned branch of figure 6, dynamic equation for the filter capacitor in each phase is

$$
C_{f m} \frac{d v_{f m}}{d t}=i_{f m}
$$

Transforming (10) with a $m^{\text {th }}$ order SRF

$$
\frac{d}{d t}\left[\begin{array}{c}
v_{f m d}^{m} \\
v_{f m q}^{m}
\end{array}\right]+\left[\begin{array}{cc}
0 & m \omega \\
-m \omega & 0
\end{array}\right]\left[\begin{array}{c}
v_{f m d}^{m} \\
v_{f m q}^{m}
\end{array}\right]=\frac{1}{C_{f m}}\left[\begin{array}{c}
i_{f m d}^{m} \\
i_{f m q}^{m}
\end{array}\right] .
$$

In steady-state, assuming accurate phase lock of the PLL, $v_{f m d}^{m}$ and $v_{f m q}^{m}$ are transformed into dc quantities. So,

$$
\frac{d v_{f m d}^{m}}{d t}=\frac{d v_{f m q}^{m}}{d t}=0,
$$

which after substitution in (11) and algebraic operation gives

$$
\begin{aligned}
v_{f m d}^{m} & =\frac{i_{f m q}^{m}}{m \omega C_{f m}} \\
v_{f m q}^{m} & =-\frac{i_{f m d}^{m}}{m \omega C_{f m}} .
\end{aligned}
$$


Table 2. System parameters.

\begin{tabular}{lcccccc}
\hline $\begin{array}{l}\text { Sampling } \\
\text { interval } \\
\left(T_{s}\right)\end{array}$ & $\begin{array}{c}\text { VSI } \\
\text { Switching } \\
\text { frequency } \\
\left(f_{c}\right)\end{array}$ & $\begin{array}{c}\text { VSI } \\
\text { dc link } \\
\text { voltage } \\
\left(V_{d c}\right)\end{array}$ & $\begin{array}{c}\text { Load } \\
\text { inductance } \\
\left(L_{d}\right)\end{array}$ & $\begin{array}{c}\text { Load } \\
\text { resistance } \\
\left(R_{d}\right)\end{array}$ & $\begin{array}{c}\text { PCC } \\
\text { voltage } \\
(1-1, \mathrm{rms})\end{array}$ & $\begin{array}{c}\text { System } \\
\text { frequency } \\
(f)\end{array}$ \\
\hline $100 \mu \mathrm{s}$ & $10 \mathrm{kHz}$ & $80 \mathrm{~V}$ & $10 \cdot 0 \mathrm{mH}$ & $30 \Omega$ & $415 \mathrm{~V}$ & $50 \mathrm{~Hz}$ \\
\hline
\end{tabular}

As the filter currents are anyway measured for current control, this allows for estimation of filter capacitor voltages without additional measurement overheads. This scheme yields strictly accurate results in the steady-state. But harmonics, being periodic functions, are steady-state phenomena. Consequently, filtering performance is not affected.

\section{Simulation results}

Performance of closed loop system is simulated for both pure sinusoidal and distorted PCC voltage cases. Multi-rate simulation is carried out using $2 \mu$ s sampling interval for the continuous time plant and $100 \mu$ s for the discrete-time controller. System parameters used are indicated in table 2. Passive filter parameters used in simulation are as detailed in table $1 . L_{d}$ and $R_{d}$ are the filter inductance and load resistance on the dc side of the rectifier load.

Performance of the SHAF is tested under various operating conditions. Specifically, filter performance under distorted PCC voltages and de-tuning of passive filter is investigated and reported.

\subsection{Case A: PCC voltage without distortion}

Figure $7 \mathrm{a}$, shows the simulated results for pure sinusoidal PCC voltage. Figure $7 \mathrm{~b}$ shows the source and load current harmonic spectra. It is seen that the compensated $5^{\text {th }}$ and $7^{\text {th }}$ harmonic source currents are within IEEE 519-1992 limits. The $11^{\text {th }}$ and $13^{\text {th }}$ harmonic currents are not affected.
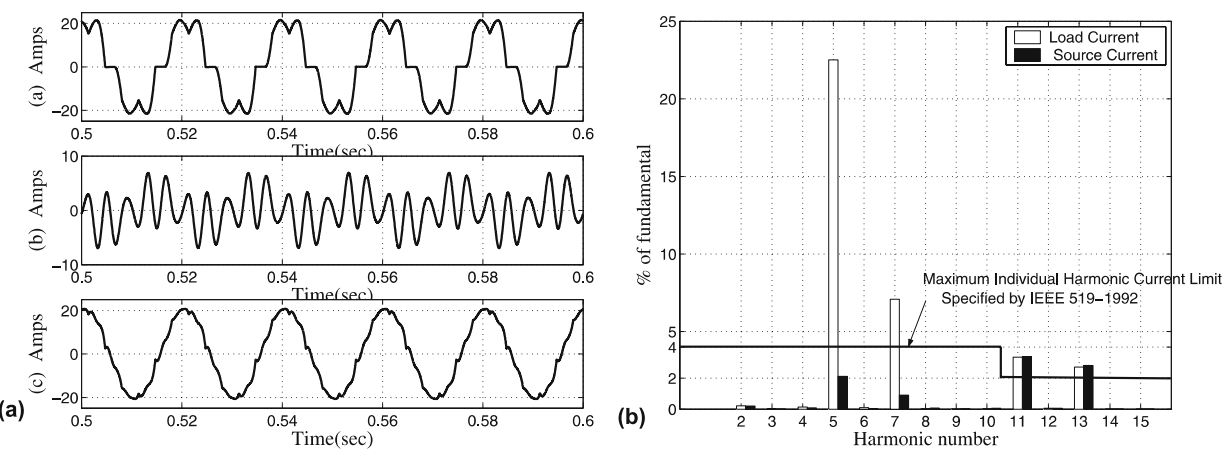

Figure 7. Case A: Simulation results and harmonic analysis. (a) Line currents: Upper trace- Load, middle- Filter, bottom- Source. (b) FFT of Load and Source currents. 

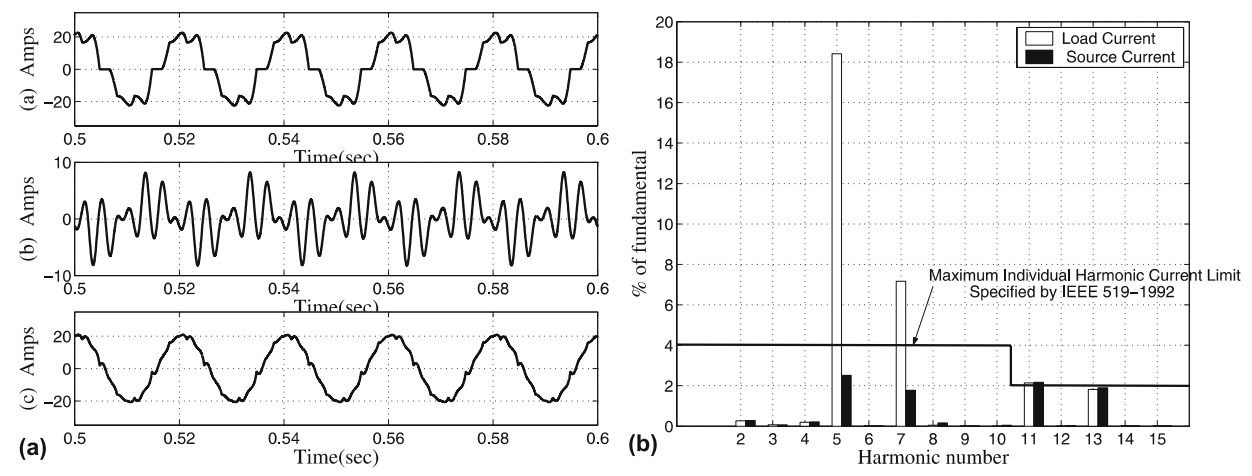

Figure 8. Case B: Simulation results and harmonic analysis. (a) Line currents: Upper trace- Load, middle- Filter, bottom- Source. (b) FFT of Load and Source currents.

\subsection{Case B: PCC voltage with distortion}

Figure 8 a shows the simulated results for distorted PCC voltage having $5 \%$ of $5^{\text {th }}$ and $4 \%$ $7^{\text {th }}$ harmonics. Figure $8 \mathrm{~b}$ shows the FFT analysis of source and load currents. The $5^{\text {th }}$ and $7^{\text {th }}$ harmonic source currents are reduced within IEEE 519-1992 limits. Comparison with Case A reveals a slight increase in the overall percentage of $5^{\text {th }}$ and $7^{\text {th }}$ harmonics in the compensated source currents. This is attributed to the slight deviation from the assumption made in deriving equation (4). However, as the PCC voltage distortion considered is realistic, this is not expected to further aggravate filtering performance with the proposed scheme. Also, the $11^{\text {th }}$ and $13^{\text {th }}$ harmonic currents are not affected by compensation.

\subsection{Case C: De-tuned passive filter}

Here, the extreme adverse operating conditions are considered. It is known that the passive filter component values are liable to drift due to variations in temperature and other environmental parameters. In such cases, the resonant frequency of the passive filter branches digress from their original value, causing deterioration in the filtering performance. So, in this case de-tuning of both passive filter branches is considered along with distortion in the supply voltage. Figure 9a shows the simulated results for this case. The extent of de-tuning, or shift in the resonant frequency of the passive branches, considered is $+5 \%$. Accordingly, the resonant frequency of the $5^{\text {th }}$ harmonic filter is shifted from $250 \mathrm{~Hz}$ to $238 \mathrm{~Hz}$. For the $7^{\text {th }}$ harmonic filter, the corresponding shift is from $350 \mathrm{~Hz}$ to $332 \mathrm{~Hz}$. Figure $9 \mathrm{~b}$ shows the FFT analysis of source and load currents. The $5^{\text {th }}(3.92 \%)$ and $7^{\text {th }}(1.93 \%)$ harmonics in the source current are again seen to be compensated within IEEE 519-1992 limits. There is a marginal increase in the $11^{\text {th }}$ and $13^{\text {th }}$ harmonics. However, these harmonics are not within the scope of the present compensation objective.

\section{Experimental results and discussions}

The proposed strategy has been experimentally tested on a load-fed from $415 \mathrm{~V}, 3$-phase, $50 \mathrm{~Hz}$ supply. The load comprised a 3-phase, diode-bridge rectifier feeding a resistive load through an inductive filter. Parameters of the load are provided in table 2. A two-level, low power (400 VA) VSI with MOSFET switches was used. DC link voltage of the VSI was 

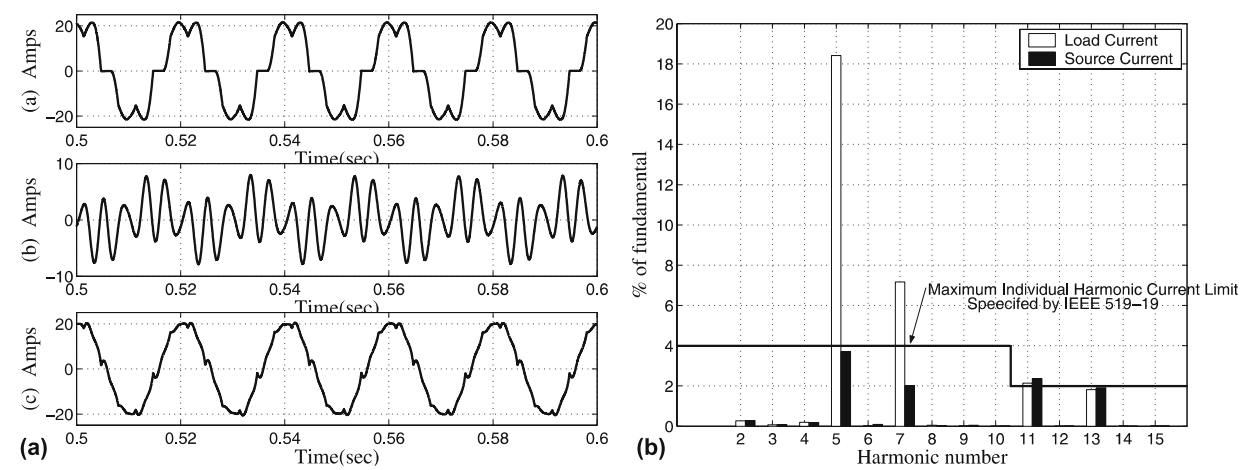

Figure 9. Case C: Simulation results and harmonic analysis. (a) Line currents: Upper trace- Load, middle- Filter, bottom- Source. (b) FFT of Load and Source currents.

controlled in an ad-hoc manner using a diode rectifier. The entire control algorithm, including harmonic extraction, PLL and VSI switching command generation, was realized on a DSP platform, using TMS320F240 processor. Hall effect current sensors were used to sense the filter and load currents. Experimental waveforms were recorded with a $500 \mathrm{MHz}$, 4-channel Digital Storage Oscilloscope (Yokogawa). Harmonic spectra are obtained using standard FFT routines available in MATLAB. The experimental set-up is shown in figure 10.

Using this set-up, the performance of the SHAF was tested under different rectifier loading conditions. Due to practical constraints, neither arbitrary distortion in the PCC voltage nor de-tuning of the passive filter could be created. Figure 11a shows the relevant waveforms under light load conditions. The filter current, load current and source current waveforms are shown. Here, the fundamental load current is $5 \mathrm{~A}$ (rms). Figure 12a shows the same variables under heavy load, the fundamental component of the load current being $12 \mathrm{~A}$ (rms). The source current waveforms in both these cases are apparently sinusoidal.

Figures $11 \mathrm{~b}$ and $12 \mathrm{~b}$ show the harmonic spectra of the source and load currents. The load current spectrum is same as that of the source current in the absence of compensation. The $5^{\text {th }}$

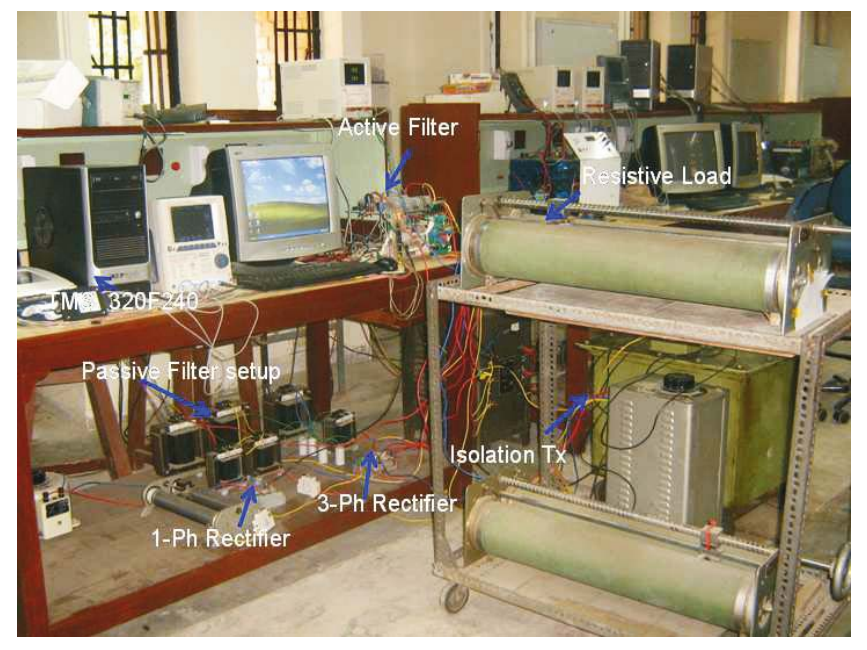

Figure 10. Experimental set-up. 

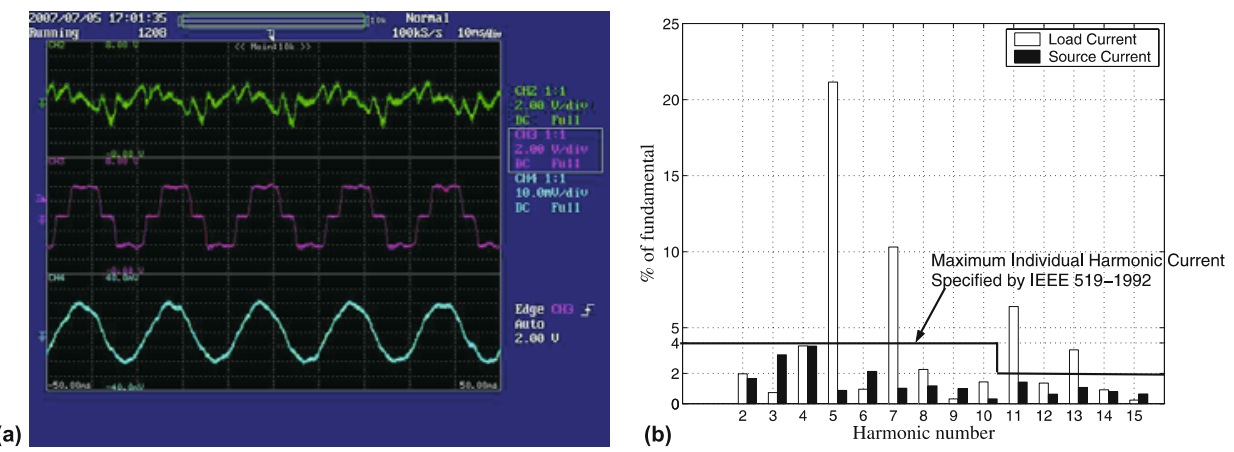

Figure 11. Experimental results on light load and comparative harmonic analysis. (a) Upper traceFilter current, middle- Load current, bottom- Source current. (b) FFT Comparison of Load and Source currents.

$(1.85 \%)$ and $7^{\text {th }}(1.67 \%)$ source harmonic currents are reduced within recommended limits of IEEE 519-1992. The $11^{\text {th }}$ and $13^{\text {th }}$ harmonics in source currents are also seen to reduce, although these were not specifically targeted for compensation. A possible reason for this could be the restricted numerical precision of the digital controller hardware. The low pass filters used for harmonic extraction could not be realized with as low corner frequencies as was possible in simulation, which uses double-precision arithmetic. As a result, reference current for the $7^{\text {th }}$ harmonic controller has fractional content of the domain-transformed $11^{\text {th }}$ and $13^{\text {th }}$ harmonic load currents. Controller bandwidth being sufficiently larger, these currents appear in the filter output, although with considerable phase shift. As the absolute magnitudes of these harmonics are quite low, all second order effects in the experimental realization also contribute.

But if higher harmonics are additionally sought to be compensated, the proposed control scheme can be successfully used with additional passive tuned branches. Elements of these additional passive branches, being tuned at higher frequencies, will be smaller than the ones considered here, both in terms of component values and current rating. Provided hardware bandwidth of the VSI is adequate, its rating does not need to be changed. Since the VSI is

(a)

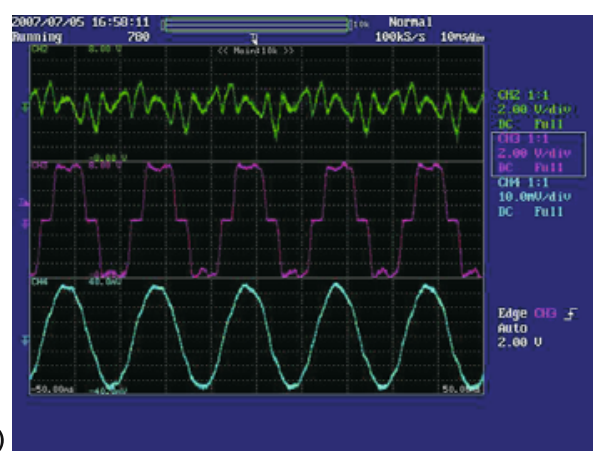

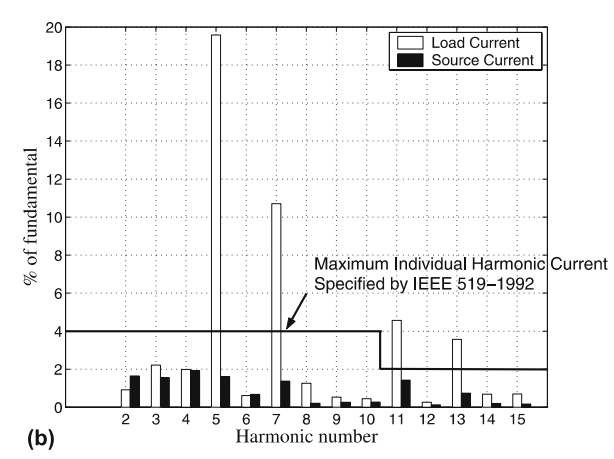

(b)

Figure 12. Experimental results on heavy load and comparative harmonic analysis. (a) Upper traceFilter current, middle- Load current, bottom- Source current. (b) FFT Comparison of Load and Source currents. 
the overbearing cost-intensive component, incremental cost for upgrading filter bandwidth is significantly low. Without deviating into economic analysis, the presented method provides a modular harmonic filtering solution, with the least cost of upgrades, coupled with excellent filtering performance.

\section{Conclusion}

A Multi-band SHAF scheme with a very low power VSI is shown to be an attractive harmonic compensation solution. This ensures a reliable yet low cost solution without sacrificing any performance advantage. Using a novel estimation scheme and standard control methods, need for measurement of branch currents and internal voltages of individual passive branches is obviated. Since the PCC voltages, load and VSI currents are the only measurements required, significant reduction in sensor count is achieved. This sensor overhead does not increase as the SHAF is augmented with additional frequency bands leading to decreasing marginal costs with every frequency upgrade. The paper reports the complete analytical design procedure of the passive filter and controller parameters. It is verified by simulation that the proposed approach is immune to parameter variations in the passive filter and distortions in the supply voltage. Proposed control algorithm for the special case of a dual-band compensator was verified experimentally on a full-scale non-linear load. Experimental results obtained conclusively prove excellent filtering performance and complete agreement with the recommendations of IEEE 519-1992. Although specifically applied to a dual-band compensator, the control algorithm presented here does not pose any restriction on the frequency band of the SHAF. So, this method is adequately general for a multi-band SHAF of any arbitrary frequency band, subject to available hardware bandwidth. The solution presented is totally modular and allows for the easiest and least expensive retrofit procedure for upgrading filtering performance and reliability of any existing passive filter or SHAF with limited bandwidth.

The authors would like to thank the Ministry of Information Technology, Govt. of India, and Centre for Development of Advanced Telematics, Thiruvananthapuram, for the infrastructure support made available through the NaMPET project. Thanks are also due to Mr Amit Basu, Mr S K Kole and Mr Nandkishor for all the support during hardware fabrication and experimental work.

\section{References}

Akagi H $1996 \mathrm{New}$ trends in active filters for power conditioning. IEEE Transactions on Industry Applications 32(6): 1312-1322

Akagi H 1997 Control strategy and site selection of a shunt active filter for damping of harmonic propagation in power distribution systems. IEEE Transactions on Power Delivery 12(1): 354-363

Bhattacharya S, Divan D M, Banerjee B 1991 Synchronous Reference Frame Harmonic Isolator Using Series Active Filter, Proceedings of $4^{\text {th }}$ EPE, Florence, Italy, 3: 30-35

Chen Q, Chen Z, McCormick M, Dinu A 2004 An Analysis and Simulation of A New Hybrid Power Filter. Proceedings of the Second International Conference on Power Electronics, Machines and Drives PEMD 2004, Edinburgh, UK 
Chen Z, Blaabjerg F, Padersen J K 2004 Harmonic Resonance Damping with a Hybrid Compensation System in Power System with Dispersed Generation. Proceedings of Thirty Fifth IEEE Power Electronics Specialists' Conference PESC 04, Aachen, Germany

Cheng P-T, Bhattacharya S, Divan D M 1999 Line harmonics reduction in high-power systems using square-wave inverters based dominant harmonic active filter. IEEE Transactions on Power Electronics 14(2): 265-272

Dugan R C, McGranaghan M F, Santoso S, Beaty H W 2003 Electrical Power Systems Quality, McGraw-Hill, 2nd ed.

Fujita H, Akagi H 1991 A practical approach to harmonic compensation in power systems-series connection of passive and active filters, IEEE Transactions on Industry Applications 27(6): $1020-1025$

Inzunza R, Akagi H 2005 A 6.6-kV transformerless shunt hybrid active filter for installation on a power distribution system. IEEE Transactions on Power Electronics 20(4): 893-900

Kaura V, Blasko V 1997 Operation of phase locked loop system under distorted utility conditions, IEEE Transactions on Industry Applications 33(1): 58-63

Longhui W, Fang Z, Pengbo Z, Hui L, Zhaoan W 2007 Stability Analysis and Controller Design of Hybrid Compensation System with Parallel Active Power Filter and Parallel Capacitors, Proceedings of IEEE Power Electronics Specialists' Conference PESC 07, Orlando, USA

Park S, Han S-B, Jung B-M, Choi S-H, Jeong H-G 2000 A Current Control Scheme Based on Multiple Synchronous Reference Frame for Parallel Hybrid Active Filter, Proceedings of Power Electronics and Motion Control Conference 2000, The Third International PIMEC 2000, Beijing, China

Peng F Z 1998 Application issues of active power filters. IEEE Industry Applications Magazine 21-30

Rastogi M, Mohan N, Edris A-A 1995 Hybrid-active filtering of harmonic currents in power systems. IEEE Transactions on Power Delivery 10(4): 1994-2000

Routimo M, Salo M, Tuusa H 2004 Wideband Harmonic Compensation with a Voltage-Source Hybrid Active Power Filter, Proceedings of Nineteenth Annual IEEE Applied Power Electronics Conference APEC 2004, Anaheim, California, USA

Sensarma P S, Padiyar K R, Ramanarayanan V 2000 A Comparative Study of Harmonic Filtering Strategies for a Shunt Active Filter, Proceedings of IEEE Industry Applications Society Annual Meeting, Rome, Italy

Srianthumrong S, Akagi H 2003 A medium-voltage tranformerless AC/DC power conversion system consisting of a diode rectifier and a shunt hybrid filter. IEEE Transactions on Industry Applications 39(3): 874-882

Zhai X, Zhuo F, Duan R, Lei W, Zhang P, Wang Z 2006 Development of a Parallel Hybrid Power Filter with Respective Harmonic Compensation Method, Proceedings of Twenty First Annual IEEE Applied Power Electronics Conference 